\title{
RETINAL NERVE FIBER LAYER DEFECT IN A PATIENT WITH HEALTHY NEURORETINAL RIM
}

\author{
Luciana P. Malta de Alencar, Fábio Konno, Ana Carolina Raiza, Remo Susanna Jr.
}

\section{INTRODUCTION}

Ophthalmoscopic scrutiny of the nerve fiber layer was first advocated by Hoyt, ${ }^{1}$ over 30 years ago. In the following years, the importance of localized defects of the retinal nerve fiber layer (NFL) for the diagnosis of glaucoma has been demonstrated in many studies. The evaluation of the NFL, though relatively difficult and subjective, has become routine in the common ophthalmic examination. ${ }^{2}$ We present a case of an isolated NFL defect with different possible etiologies.

\section{DESCRIPTION OF THE CASE}

A 45-year-old white man with diabetes mellitus and systemic arterial hypertension was referred for routine consultation. Ophthalmological examination revealed a best corrected visual acuity of 20/20 in both eyes (OU). Slitlamp biomicroscopy and gonioscopy were unremarkable. The patient presented with intraocular pressures (IOP) that ranged from 13 to $16 \mathrm{mmHg} \mathrm{OU}$, which was confirmed on modified diurnal curve monitoring. Cup-disk ratios were $0.6 \mathrm{OU}$, with healthy neural rims, without evidence of thinning, notching, or localized pallor. However, there was an evident inferior-temporal wedge-shaped defect of the NFL on the left eye (Figure 1). There were no signs of diabetic

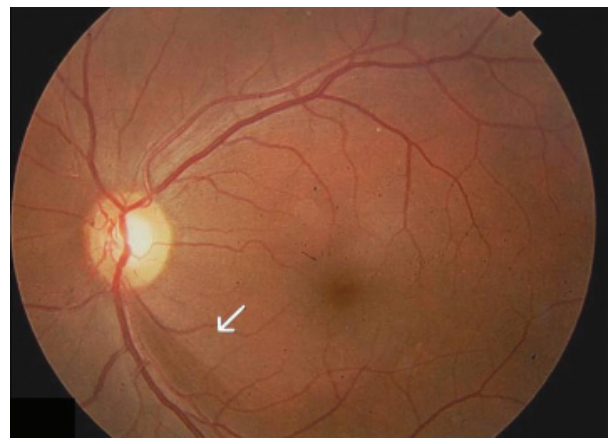

Figure 1 - The inferior localized retinal nerve fiber layer defect (arrow)

Ophthalmology Department, Hospital das Clínicas, São Paulo University Medical School - São Paulo/SP, Brazil.

Email: alencarluciana@yahoo.com.br retinopathy OU. The patient had an unremarkable family history.

Subsequent psychophysical testing confirmed a superior initial defect on the visual field of this eye with conventional achromatic perimetry (SITA Standard 24-2) (Figure 2) and frequency doubling perimetry (Figure 3). Shortwavelength perimetry was normal OU. The nerve fiber analysis with scanning laser polarimetry (GDx VCC - Laser Diagnostic Technologies, San Diego, CA) showed nerve fiber layer regional inferior defects $\mathrm{OU}$, which were more evident in the left eye (Figure 4), both on the reflectance image and on the retardation image. Confocal scanning laser ophthalmoscopy (HRT - Heidelberg Retina Tomograph,

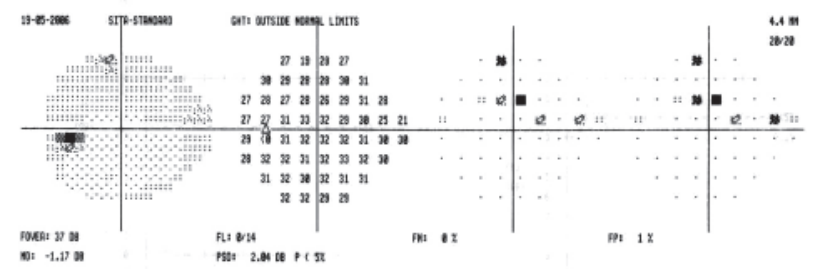

Figure 2 - Visual field of the same eye above, showing the superior defect

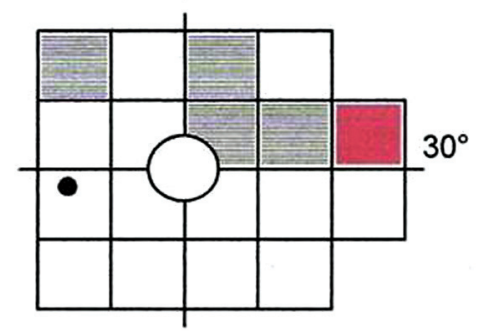

Figure 3 - Frequency doubling perimetry showing the superior defect
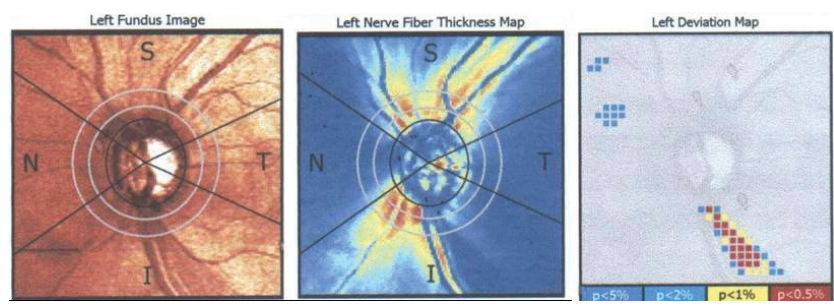

Figure 4 - GDx VCC deviation map with a correspondent localized inferior defect in the nerve fiber layer 
Heidelberg Engineering $\mathrm{GmbH}$, Germany) was borderline in the left eye, and optical coherence tomography (Stratus OCT - Carl Zeiss Meditec) was unremarkable. Except for the GDx, all tests were normal for the right eye.

\section{DISCUSSION}

At times when pre-perimetric diagnosis of glaucoma is the goal, the search for the subtle signs of damage in the NFL is of utmost importance. Retinal nerve fiber layer defects have been shown to be among the earliest signs of glaucomatous damage, and they can indeed precede visual field defects. ${ }^{3,4}$ They are especially helpful for early glaucoma diagnosis and in eyes with small optic disks. The localized wedge-shaped defect of the NFL is usually seen in association with notching of the neuroretinal rim, vertical enlargement of the cup, or following disk hemorrhages. Nevertheless, in early glaucoma, bundle defects in the NFL may not be associated with neuroretinal rim thinning because the initial damaged NFL is located in the deep retinal layers. Hence, typical wedge-shaped defects can be found in disks with normal appearance. ${ }^{5-7}$

Since NFL defects are not present in normal eyes, they always indicate an abnormality. Although typically occurring in about $20 \%$ of all eyes with glaucoma, they are not pathognomonic and can also be found in other ocular diseases, such as optic disk drusen, ischemic retinopathies with cotton-wool spots, toxoplasmotic retinochoroidal scars, long-standing papilledema, or optic neuritis due to multiple sclerosis. The incidence is higher in normal-tension glaucoma than in the other forms, which makes the differential diagnosis somewhat difficult.

Some authors have shown that NFL defects may be a common finding in diabetic patients with early diabetic retinopathy, and one of the risk factors is concomitant high blood pressure. ${ }^{8}$ Retinal nerve fiber layer thickness has been found to decrease with the development of diabetic retinopathy and with impairment of metabolic regulation. ${ }^{9}$ Cotton-wool spots are frequently a feature of systemic arteriolar disease, most commonly hypertension, diabetes, and collagen vascular disease; they represent infarcts at the nerve fiber layer. Cotton-wool spots have been described to be followed in some patients by localized NFL defects, with and without associated visual field defect. ${ }^{10,11}$

This case report describes a middle-aged patient with an isolated arcuate defect within the nerve fiber layer and visual field, without recognizable abnormality of the optic disk or cup. Although NFL raises suspicion for glaucoma and prompts a careful follow-up, other possible systemic reasons for localized retinal ischemia must also be considered.

\section{REFERENCES}

1. Hoyt WF, Schlicke B, Eckelhoff RJ. Fundoscopic appearance of a nervefibre-bundle defect. Br J Ophthalmol. 1972;56:577-83.

2. Fingeret M, Medeiros FA, Susanna R, Jr., Weinreb RN. Five rules to evaluate the optic disc and retinal nerve fiber layer for glaucoma. Optometry. 2005;76:661-8.

3. Sommer A, Quigley HA, Robin AL, Miller NR, Katz J, Arkell S. Evaluation of nerve fiber layer assessment. Arch Ophthalmol. 1984;102:1766-71.

4. Sommer A, Katz J, Quigley HA, Miller NR, Robin AL, Richter RC, et al. Clinically detectable nerve fiber atrophy precedes the onset of glaucomatous field loss. Arch Ophthalmol. 1991;109:77-83.

5. Jonas JB, Dichtl A. Evaluation of the retinal nerve fiber layer. Surv Ophthalmol. 1996;40:369-78.

6. Jonas JB, Fernandez MC, Sturmer J. Pattern of glaucomatous neuroretinal rim loss. Ophthalmology. 1993;100:63-8.
7. Jonas JB, Schiro D. Localised wedge shaped defects of the retinal nerve fibre layer in glaucoma. Br J Ophthalmol. 1994;78:285-90.

8. Chihara E, Matsuoka T, Ogura Y and Matsumura M, Retinal nerve fiber layer defect as an early manifestation of diabetic retinopathy, Ophthalmology. 1993;100:1147-51

9. Ozdek S, Lonneville YH, Onol M, Yetkin I, Hasanreisoglu B. Assessment of nerve fiber layer in diabetic patients with scanning laser polarimetry. Eye. 2002;16:761-5.

10. Chaum E, Drewry RD, Ware GT, Charles S. Nerve fiber bundle visual field defect resulting from a giant peripapillary cotton-wool spot. J Neuroophthalmol. 2001;21:276-7.

11. Chihara E, Honda Y. Topographic changes in the optic disc in eyes with cotton-wool spots and primary open-angle glaucoma. Graefes Arch Clin Exp Ophthalmol. 1991;229:13-8. 